\title{
Investigation of Synbiotic Effect in Thai Night Shift Workers Identified by Epworth Sleepiness Scales
}

\author{
Phuttharaksa Phumcharoen ${ }^{\mathrm{a}, \mathrm{b}}$, Wichian Sittiprapaporn ${ }^{\mathrm{a},{ }^{*}}$ \\ ${ }^{a}$ Neuropsychological Research Laboratory, Department of Anti-Aging and Regenerative Science, School of Anti-Aging and Regenerative \\ Medicine, Mae Fah Luang University, Bangkok 10110, Thailand \\ ${ }^{b}$ Department of Anti-Aging and Regenerative Medicine, School of Anti-Aging and Regenerative Medicine, Mae Fah Luang University, \\ Bangkok 10110, Thailand \\ Corresponding author: *drwichian.s@gmail.com
}

\begin{abstract}
Many sleep disorders are characterized by excessive sleepiness. As a type of circadian rhythms sleep-wake disorder, shift work sleep disorder consists of insomnia or excessive drowsiness caused by a recurrent task schedule that crosses with usual sleep hours. Night-shift workers have a disrupted circadian rhythm, indicating less overall sleep time than evening and day workers. Probiotic supplementation has been found to improve subjective sleep quality, associated with balancing gut microbiota. The objective was to investigate the sleeping habits of night shift workers after treating with a synbiotic supplement. Eleven excessive drowsiness among night-shift workers was included in this study. Epworth Sleepiness Scale assessed each participant. For 8 weeks, all participants were given a synbiotic supplement containing 7 probiotics and 3 prebiotics once a day. The Epworth Sleepiness Scales of night shift workers who participated in the pre-intervention showed that most of the night shift workers were mild excessive daytime sleepiness, while fewer night shift workers showed moderate excessive daytime sleepiness. It was found that most of the night shift workers were moved to lower normal daytime sleepiness, while the less of night shift workers showed higher normal daytime sleepiness. It showed a significant improvement of the Epworth Sleepiness Scales $(p=\mathbf{0 . 0 0 3})$. Although these data are preliminary, they may not reflect all of the night shift workers' sleep propensities after treatment with synbiotics. It requires more research to be conducted in a wider scale.
\end{abstract}

Keywords - Sleep; shift worker; excessive sleepiness; epworth sleepiness scale; synbiotic.

\section{INTRODUCTION}

The human body is a huge source of microorganisms and viruses, archaea, unicellular eukaryotes, and bacteria, collectively mentioned as the microbiota. Despite the fact that bacteria colonize almost all surfaces of the human body, the digestive tract is the most colonized, with microbial density in the large intestine reaching $10^{11}-10^{12}$ per $\mathrm{mL}$ and over 1,000 species. In any case, fundamental between individuals diverse in microorganisms piece exist [1]. Although common species have been discovered among humans by $16 \mathrm{~S}$ and shotgun sequencing, there is a wide range of variation in many common species categories, ranging from 12-to-2,200-fold. This variability results from the accumulated impact of host hereditary qualities, aging, geographic areas, sex, lifestyle, and diet; many of these factors are combined. Balanced gut microbiota is essential for physiology, and its alteration has been linked to a variety of disorders. Obesity, for example, is linked to a decrease in Bacteroidetes and an increase in Firmicutes. In addition, previous research reveals that lack of sleep is linked to gut dysbiosis, and that circadian disturbance affects the intestinal microbiota. Furthermore, they produce end products that respond to sleep deprivation are capable of inducing exhaustion. A recent study showed that probiotic supplementation could improve subjective sleep quality associated with balancing gut microbiota [1], [2].

Working in shifts as defined by the International Labor Organization (ILO) in Geneva in 1990 as "a method of organization of working time in which workers succeed one another at the workplace so that the establishment can operate longer than the hours of work of individual workers" at various times of the day and night [2]. Shift work can be done on a full-time or part-time basis in every country across the world. The European Council Directive 93/104 (1993) declared that after three years, "concerning certain aspects of the organization of working time, shift work shall mean any methods of organizing work in shifts, whereby workers 
succeed each other at the same workstations according to a certain pattern. Shift worker shall mean any workers whose work schedules are part of shift work." Shift work has been defined as any schedule of regular working time other than the usual daytime (07:00 - 08:00 a.m. to 05:00 - 06:00 p.m.) in the published studies. Approximately 15-20 percent of workers in developed countries work shifts. Morning, evening, and night shifts are the most common work patterns.

On the other hand, shift rotation is characterized by fluctuating and unpredictable work schedules dependent on the employer's demands [1], [2]. In 2010, both Occupational Health and National Health Interview Survey showed that shift workers in the United States accounted for $29 \%$ of the total workforce. Notably, the most noticeable prevalence of shift work was seen in the food preparing and serving industries, which accounted for over 63 percent of the total. According to previous research, night shift workers may have experienced issues with their level of cognitive impairment. Shift workers' circadian rhythms were misaligned.

Furthermore, numerous shift workers' circadian rhythms were disrupted due to their night shift schedule [2]. Night shift work time, which typically ranged from 21:00 to 08:00, was linked to a continual reduction in sleep time ( $<7$ hours for every 24 hours). This leads to an increase in sleepiness and a decrease in capacity. Additionally, workers on night shifts were more likely than those who worked during the day to have excessive sleepiness during arousal hours [3]. These shifts in circadian rhythm were linked to increased drowsiness, poor function, and sleep fragmentation among night-shift workers.

Additional research established that night-shift workers' circadian rhythms were misaligned, resulting in less overall sleep than evening and day employees. Furthermore, a recent study shows that night-shift workers were more impaired on alertness and performance than day shifts [4]. Excessive sleepiness was affecting the activities of everyday life for almost 45 percent of night-shift workers. Nevertheless, not everyone who works shifts develops shift work sleep disorder. Changes in sleep and circadian physiology can induce shift work sleep disruption. Shift workers with extreme sleepiness throughout their shifts and clinically severe disrupted sleep that interfered with social, occupational, or other areas of their lives were also at risk for shift work sleep disorder [5]. Circadian oscillation disruption in humans can be caused by a variety of events, including shift employment and traveling across time zones. Furthermore, which refers to this recent study, many factors were contributing to disturbed circadian oscillations. Dampened circadian oscillations, which could be due to chronodisruption, might have resulted from gene polymorphism, desynchronisation of the environment (shift work, sleep restriction, behavioral), including physiological aging [6].

Shift work has been linked to a higher incidence of metabolic disorders, including type 2 diabetes, obesity, and metabolic syndrome [7]. Recent studies suggest that disruption of the circadian clock was a key supporting factor in various pathological conditions such as sleep disorders, cancer, gastrointestinal system, cardiovascular disease, and metabolic syndrome [8]-[10]. A lot of studies have proved higher rates of various brain disorders such as neurodegenerative disease in shift workers [11], [12].
Additionally, circadian oscillations have strong control over immunological function, and disruption of circadian oscillations may have deleterious consequences. One common feature of disorders associated with a disturbance of circadian oscillations is that they appear to be triggered by inflammatory processes [13].

Previous studies found that circadian rhythm disturbance in shift workers led to the change in the abundance of the gut microbiome. The relative richness of Actinobacteria and Firmicutes was raised, while those of Bacteroidetes decreased when working night shifts compared to day shifts. Likewise, shift works were reported that they cause the alteration of one's sleep patterns and eating habits, leading to the change in the composition of the gut microbiota. Circadian rhythm disruption causes gut dysbiosis, contributing to an inflammatory state associated with shift work [14]. On the other hand, several studies have reported the benefits of probiotics on sleep quality, mood, and cognitive functions. Recent meta-analytic studies have shown probiotics supplementation could significantly improve some efficacy in perceived sleep quality, measured using decreased Pittsburgh Sleep Quality Index (PSQI) score relative to baseline [14].

Shift work sleep disorder (SWSD) usually complains about excessive sleepiness and/or insomnia related to circadian misalignment. Difficulty sleeping due to shift employment is one type of circadian rhythm. People with CRSDs have trouble keeping up with external factors like duties in society or at work. External circumstances push a person's schedule that contradicts their internal clock. Insomnia or excessive drowsiness are symptoms of shift work sleep disorder, a type of CRSD caused by a typical work schedule that corresponds with the regular sleep cycle. The Epworth Sleepiness Scale (ESS) is used to assess the sleep propensity of adult patients who have sleep issues and are sleepy throughout the day.

The ESS is used to diagnose obstructive sleep apnea, periodic limb movement disorder, narcolepsy, and idiopathic hypersomnia, as well as other sleep disorders. The ESS can be used to assess sleep proclivity in eight unique circumstances based on examining the subject's retrospective descriptions of napping habits. These eight scenarios are regularly encountered in daily life. A critical component of each patient's examination is the severity of their excessive daytime drowsiness. The test-retest reliability of the ESS is quite good. Additionally, its eight circumstances exhibit a high degree of internal consistency. In distinguishing normal subjects from patients with various sleep disorders, the ESS scores were found to be substantially linked with the severity of excessive daytime sleepiness [11-[14]. In this study, the bioinformatics of synbiotic supplement influence on sleep propensities (SPs) in night shift workers working in an industrial pharmaceutical sector in Thailand was investigated in several scenarios using the ESS. The present study aimed to determine the effect of a synbiotic supplement on daytime sleepiness in night shift employees.

\section{MAterials AND METHOD}

\section{A. Participants}

Eleven participants aged between 25-60 years old who were night shift workers participated in this study. All participants were interviewed and screened according to the 
inclusion criteria, including a night shift worker who works at least five nights each month. Each shift was $\leq 12 \mathrm{hrs}$. and $\geq 6$ hrs., between 24.00 and 05.00 hours. Shift work has a threemonth minimum requirement. All of the participants met the ESS's criteria for extreme sleepiness. The ESS score were $\geq$ 11 before assigning to participate in the study. Several criteria where any participants were excluded, such as self-reporting of main medical diseases including kidney failure, liver cirrhosis, cardiac failure, and cerebrovascular diseases. They were excluded if they showed mental or brain problems and a history of head injury or hearing or vision difficulties.

Participants who had a history of alcohol or drug misuse and any central nervous system active drugs or substances that could produce sedation the week before the trial were excluded. The normal 24-hour coffee consumption of $>750$ $\mathrm{mg}$, the use of other vitamins and botanicals throughout this research. Antibiotics were used in this investigation and an unfavorable reaction to a probiotic or prebiotic supplement in the past were all considered as reasons to exclude participants from the study. After all, participants received information about the research. They were given signed consent before participation in the study. This study was carried out under the Helsinki Declaration. The local institutional review board also approved the study of Mae Fah Luang University, Thailand.

\section{B. Synbiotic preparation}

Lactomason Korea Co., Ltd. produced the synbiotics utilized in this investigation. Lactobacillus rhamnosus, Lactobacillus paracasei, Lactobacillus reuteri, Lactobacillus salivarius, and Bifidobacterium lactis, Bifidobacterium breve, and Bifidobacterium longum were used as synbiotics, respectively. All probiotics in synbiotics have a colonyforming unit count of $25 \times 10^{9}$ per gram. They also contained 4 grams of inulin, 2 grams of fructooligosaccharide, and 2 grams of galactooligosaccharide.

\section{Intervention}

Synbiotics were administered to all the participants in the form of aluminum foil sachets. For eight weeks, they were supposed to drink one sachet every day. Everyone was notified regularly via phone. However, during the course of the investigation, they did not allow the participants to use any additional dietary supplements or botanicals. One sachet of synbiotic supplement (manufactured by Lactomason Korea) comprises the following bacteria: Bifidobacterium breve $(5 \times$ $\left.10^{9} \mathrm{CFU}\right)$, Bifidobacterium lactis $\left(8.5 \times 10^{9} \mathrm{CFU}\right)$, Bifidobacterium longum $\left(2 \times 10^{9}\right.$ CFU), Lactobacillus paracasei $\left(3 \times 10^{9} \mathrm{CFU}\right)$, Lactobacillus reuteri $\left(0.5 \times 10^{9}\right.$ $\mathrm{CFU})$, Lactobacillus rhamnosus $\left(5 \times 10^{9} \mathrm{CFU}\right)$, Lactobacillus salivarius $\left(1 \times 10^{9} \mathrm{CFU}\right)$. A complete combination of synbiotics consists of $25 \times 10^{9} \mathrm{CFU}$ of probiotics, $4 \mathrm{~g}$ of inulin, $2 \mathrm{~g}$ of fructooligosaccharide, and $2 \mathrm{~g}$ of galactooligosaccharide. Synbiotic preparation was administered to all participants in aluminum foil sachets. For eight weeks, all participants took a sachet before bedtime and were monitored by phone regularly. They were asked to maintain their normal routines and to report any illnesses that arose throughout the trial.

\section{Excessive sleepiness}

This research applied the use of excessive sleepiness. When sleep was inappropriate, it was defined as any difficulty staying awake and/or a greater likelihood of falling asleep. In explaining symptoms of excessive sleepiness, some individuals normally use subjective expressions to describe their symptoms, including drowsiness, languor, inertness, fatigue, and sluggishness, respectively. The ESS was used to measure excessive sleepiness. Excessive sleepiness has been accurately predicted using the ESS. Excessive drowsiness was previously identified using an ESS score of 11 in prior studies.

\section{E. Epworth sleepiness scale}

Thai night shift workers' sleep propensity (SP) who worked in a pharmaceutical industrial factory in Thailand in 2019 was assessed in this research. This research utilized the ESS questionnaire as the self-administration approach established in a prior study. In 1991, Dr. Murray Johns of Melbourne's Epworth Hospital developed the ESS to assess daytime sleepiness. The ESS is an eight-question self-administered questionnaire and four levels of replies regarding a symptom's weekly frequency. The maximum score was 24 points while the lowest score was 0 . In calculating the scores, a score $\geq 11$ is considered as excessive daytime sleepiness (EDS). Moreover, if the score is greater than 20 , it is considered severe excessive daytime sleepiness. Several prior studies had successfully validated this score. The ESS was used to discriminate between sleep disordered and non-sleep disordered clinical samples in a previous study. The ESS appears to predict excessive drowsiness in everyone. To survey pervasiveness excessive drowsiness rates, a cutoff score based on an example was used. The higher the score, the higher the chance of dozing during the day and a score $\geq 11$ demonstrates excessive sleepiness (Table I). The ESS consisted of eight questions or situations that all participants were required to respond to. The following are the item numbers for the various circumstances in the questionnaire.

Scenario 1: Reading while sitting

Scenario 2: While watching television

Scenario 3: Sitting and doing nothing in a public place

Scenario 4: For an hour without stopping as a passenger in a car

Scenario 5: When circumstances allow, to lie down to relax in the afternoon.

Scenario 6: Taking a seat and conversing with someone

Scenario 7: After a non-alcoholic meal, sitting quietly.

Scenario 8: While trapped in traffic for several minutes in a car.

All participants were asked to use the following scale to select the most appropriate number for each circumstance at the start and completion of the research ( $8^{\text {th }}$ weeks $)$.

$0=$ would never doze

$1=$ slight chance of dozing

$2=$ moderate chance of dozing

$3=$ high chance of dozing 
TABLE I

DEGREE OF DOZING LEVELS AND ITS INTERPRETATION BASED ON EPWORTH SLEEPINESS SCALE

\begin{tabular}{cc}
\hline Scores & Levels of Excessive Daytime Sleepiness \\
\hline $0-5$ & Lower normal daytime sleepiness \\
$6-10$ & Higher normal daytime sleepiness \\
$11-15$ & Mild normal daytime sleepiness \\
$16-20$ & Moderate normal daytime sleepiness \\
$>20$ & Severe normal daytime sleepiness \\
\hline
\end{tabular}

\section{F. Statistical Analysis}

The descriptive analysis was used to examine the demographic data of all participants. The quantitative data were reported in terms of average and standard deviation, while qualitative data were expressed as frequency and percentage. In addition, descriptive statistics were used to perform those demographic data by utilizing characteristic measures including percentage, mean and standard deviation, respectively. Also, the inferential statistic was used to compare each situation in the ESS by Wilcoxon signed-rank test.

\section{RESULTS AND DISCUSSION}

Due to a variety of unpredictable reasons and circumstances, this investigation included just eleven participants. The mean age of participants was $51.27 \pm 1.35$. The body mass index (BMI) was determined using the average weight and height of $71.00( \pm 2.84) \mathrm{kg}$ and 165.18 $( \pm 2.08) \mathrm{cm}$. The individuals' average BMI was $26.03 \pm 0.91$ $\mathrm{kg} / \mathrm{m}^{2}$. They answered all eight situations one by one based on the standard ESS. The ESS of night shift workers participating in the pre-intervention showed that most of night shift workers were mild excessive daytime sleepiness (mild EDS: $63.63 \%$ ), while the less of night shift workers showed moderate excessive daytime sleepiness (moderate EDS: $36.36 \%$ ).

On the other hand, during the post-intervention, it was found that most of the night shift workers were moved to lower normal daytime sleepiness $(90.90 \%)$, while the less of night shift workers showed higher normal daytime sleepiness $(9.09 \%)$ (Table II). This study aimed to determine Thai night shift workers' sleep propensity (SP) in an industrial pharmaceutical factory in Thailand. The ESS of night shift workers participating in the pre-intervention showed that most night shift employees exhibited mild excessive daytime drowsiness, whereas a few exhibited significant excessive daytime sleepiness. On the other hand, during the postintervention, it was found that most of night shift workers moved to lower normal daytime sleepiness, while the less of night shift workers showed higher normal daytime sleepiness (Table II).

Furthermore, the result of this study showed a significant improvement in the ESS score $(p=0.003)$, and all situations in the ESS showed significant improvement in scores except 'S8-while trapped in traffic for several minutes in a car' (Table III). Perhaps this kind of situation required more concentration than other situations. The findings raise serious questions about the concept of sleepiness and how it is measured. The ESS ratings were all measures of situational sleep tendency. The circumstances could be generally described as "the results of eight situations in the survey" relied very much on the participants' attitudes toward them. It has already been exhibited how directions and inspiration could affect the sleep inclination in a specific circumstance.

TABLE II

LEVEL OF EXCESSIVE DAYTIME SLEEPINESS OF NIGHT SHIFT WORKERS PARTICIPATED IN THIS STUDY

\begin{tabular}{ccc}
\hline $\begin{array}{c}\text { Levels of Excessive Daytime } \\
\text { Sleepiness }\end{array}$ & Scores & Percentage \\
\hline Pre-Intervention & - & - \\
Lower normal & - & - \\
Higher normal & 7 & $63.63 \%$ \\
Mild EDS & 4 & $36.36 \%$ \\
Moderate EDS & - & - \\
Severe EDS & & \\
Post-Intervention & 10 & $90.90 \%$ \\
Lower normal & 1 & $9.09 \%$ \\
Higher normal & - & - \\
Mild EDS & - & - \\
Moderate EDS & - & - \\
Severe EDS & & \\
\hline
\end{tabular}

This study was significantly improved that the ESS score $(p=0.003)$ and all situations in the ESS showed significant improvement in scores except 'S8-while trapped in traffic for several minutes in a car' (Table III).

TABLE III

PRE- AND POST- EPWORTH SLEEPINESS SCORE IN 8 SCENARIOS BASED ON EPWORTH SLEEPINESS SCALE

\begin{tabular}{lccc}
\hline \multicolumn{1}{c}{ Scenarios } & $\begin{array}{c}\text { Pre- } \\
\text { Intervention } \\
\text { Mean } \pm \text { SD }\end{array}$ & $\begin{array}{c}\text { Post- } \\
\text { Intervention } \\
\text { Mean } \pm \text { SD }\end{array}$ & $\begin{array}{c}\boldsymbol{p} \text { - } \\
\text { value }\end{array}$ \\
\hline $\begin{array}{l}\text { S1 - Reading while } \\
\text { sitting }\end{array}$ & $2.27 \pm 0.91$ & $0.36 \pm 0.51$ & $0.009^{*}$ \\
$\begin{array}{l}\text { S2 - While watching } \\
\text { television }\end{array}$ & $2.09 \pm 1.22$ & $0.36 \pm 0.51$ & $0.011^{*}$ \\
$\begin{array}{l}\text { S3 - Sitting and doing } \\
\text { nothing in a public place }\end{array}$ & $2.09 \pm 2.04$ & $0.18 \pm 0.41$ & $0.007^{*}$ \\
$\begin{array}{l}\text { S4 - For an hour without } \\
\text { stopping as a passenger in } \\
\text { a car }\end{array}$ & $2.09 \pm 1.38$ & $0.36 \pm 0.51$ & $0.010^{*}$ \\
$\begin{array}{l}\text { S5 - When circumstances } \\
\text { allow, to lie down to relax } \\
\text { in the afternoon. }\end{array}$ & $1.91 \pm 1.38$ & $0.45 \pm 0.52$ & $0.019^{*}$ \\
$\begin{array}{l}\text { S6 - Taking a seat and } \\
\text { conversing with someone }\end{array}$ & $0.82 \pm 0.98$ & $0.00 \pm 0.00$ & $0.024^{*}$ \\
$\begin{array}{l}\text { S7 - After a non- } \\
\text { alcoholic meal, sitting } \\
\text { quietly. }\end{array}$ & $2.18 \pm 1.25$ & $0.27 \pm 0.47$ & $0.007^{*}$ \\
$\begin{array}{l}\text { S8 - While trapped in } \\
\text { traffic for several minutes } \\
\text { in a car. }\end{array}$ & $0.55 \pm 1.21$ & $0.00 \pm 0.00$ & 0.157 \\
\hline \multicolumn{1}{c}{ Total } & $14.00 \pm 2.97$ & $2.00 \pm 1.79$ & $0.003^{*}$ \\
\hline
\end{tabular}

$* \overline{\text { Indicates statistical significance at } p<0.05 \text {. All parameters were evaluated }}$ at pre-and post-treatment by Wilcoxson signed rank test.

The ESS levels representing situational sleep tendency in a specific individual were moderately consistent and could be approximated with reliability. Like the mean sleep latency in the multiple sleep latency test, the overall ESS offered a credible evaluation of the subjects' typical sleep affinity (MSLT). It is fascinating to see how chronic sleep disorders like obstructive sleep apnea or narcolepsy affect patients' daytime tiredness. Many drowsy people, for example, simply sit or lie down and relax nearly anyplace, which causes them to fall asleep. When compared to the MSLT, the ESS has a high sensitivity for detecting such people. On the other hand, the ESS identified individuals who reported being extremely drowsy in daily life but did not fall asleep during the MSLT. 
Despite the fact that the etiology of such a problem is unknown, sleep propensity (wakefulness) has been demonstrated to be very trustworthy, accurate, and valid through numerous studies [14]-[16].

It is estimated that $15-20 \%$ of the workers in industrialized countries, work as shift worker such as defensive administrations, transportations, medical services, and food planning, with demand working hours during nighttime. These kinds of night-shift work often bring about negative consequences on well-being and security. Shift workers show various degrees of transformation related to various individual factors, including working circumstances and social conditions. A lot of shift workers have a misaligned circadian clock, particularly night work plans. Excessive sleepiness, poor performance, and altered subjective sleep are manifestations of a circadian rhythm disruption in night-shift workers. As a result, their internal biological clock and sleepwake cycle become misaligned [14], [15]. The prevalence of EDS increases with age. The independent risk factors of excessive sleepiness are male sex, depressive symptoms, poor sleep quality, moderate to severe obstructive sleep apnea. Shift workers often experience excessive daytime tiredness. Excessive sleepiness has been linked to decreased cognitive, performance, and psychomotor function in previous studies. Excessive drowsiness is usually linked to a decreased sense of well-being, as well as exhaustion and poor energy levels [15]. Besides, some previous investigations indicated that excessive sleepiness significantly contributed to socioeconomic costs. Accidents caused by shift workers regularly resulted in serious injury and death, costing billions of dollars. As a result, excessive sleepiness was regarded as one of the key factors in such accidents, too [16].

According to the gut-brain axis, which involves the significant bidirectional connection between the gastrointestinal tract and the central nervous system, changing the gut microbiota environment through probiotic supplementation may help improve sleep quality. In fact, through increasing the generation of free tryptophan in the central nervous system, changes in the gut microbiome have influenced serotonin neurotransmission [17]. In turn, melatonin is formed by acetylating serotonin and methylating it, which is the regulating sleep/wake cycles hormone [18]. Previous studies demonstrated that the supplement of probiotics might play an important role in sleep quality. Probiotics supplementation in irritable bowel syndrome (IBS) in male subjects for 6 weeks could significantly increase salivary melatonin levels. Moreover, a randomized controlled trial in 40 major depressive disorders with irritable bowel syndrome patients was conducted and found that the 90-day consumption of the supplement of B. coagulans MTCC 5856 could significantly improve Modified Epworth Sleepiness Scale (mESS) compared to placebo group [19]-[21]. Likewise, our study also supported the benefit of synbiotics supplement on sleep quality in night-shift workers.

\section{CONCLUSION}

Synbiotic supplements for eight weeks significantly improved the ESS. Thai night-shift workers who work in an industrial pharmaceutical factory in Thailand were assessed using conventional criteria of excessive drowsiness. The Epworth drowsiness scale resulted in a score of 11 on the sleepiness scale. Because the situations are subjective to the participants' perspectives, they can be defined in broad terms, but not entirely. After taking a synbiotic supplement, it is possible that it does not fully reflect Thai shift workers' sleep proclivity. However, the current finding has only a small amount of data. It is important to do more research on a wider scale.

\section{ACKNOWLEDGMENT}

We are obliged to all of the subjects who took part in this research. We appreciated the National Research Council of Thailand (Grant No. 126/2561), the Thai Tobacco Authorities (Grant No. 4/2560), and the Innovation Center for Holistic Health, Nutraceuticals, and Cosmeceuticals. The authors would like to thank Mae Fah Luang University's Brain Science and Engineering Innovation Research Group for their cooperation (2019-2020). In contributing to this study, Phuttharaksa Phumcharoen conceived of the study; carried out the research; designed the study; statistically analyzed and interpreted the data; carried out a literature search; drafted manuscript preparation; obtained funding. Wichian Sittiprapaporn originated the concept; conceived of the study; carried out the research; supervised the research; interpreted the data; investigated the study; obtained funding; critical revision of the manuscript. Wichian Sittiprapaporn is the corresponding author.

\section{REFERENCES}

[1] W. Dieterich, M. Schink, and Y. Zopf, "Microbiota in the Gastrointestinal Tract," Medical sciences (Basel, Switzerland), vol. 6(4), 116, 2018. https://doi.org/10.3390/medsci6040116

[2] B.A. Matenchuk, P.J. Mandhane, and A.L. Kozyrskyj, "Sleep, circadian rhythm, and gut microbiota," Sleep Med Rev., vol. 53, 101340, 2020. https://doi.org/10.1016/j.smrv.2020.101340.

[3] A.C. Reynolds, J.L. Paterson, S.A. Ferguson, D. Stanley, K.P. Jr Wright, and D. Dawson, "The shift work and health research agenda: Considering changes in gut microbiota as a pathway linking shift work, sleep loss and circadian misalignment, and metabolic disease," Sleep Med Rev., vol. 34, pp. 3-9, 2017. https://doi.org/10.1016/j.smrv.2016.06.009.

[4] S.L. Chellappa, C.J. Morris, and F.A.J.L. Scheer, "Effects of circadian misalignment on cognition in chronic shift workers,". Sci Rep., vol. 9, 699, 2019. https://doi.org/10.1038/s41598-018-36762-w

[5] S. Jehan, F. Zizi, S.R. Pandi-Perumal, A.K. Myers, E. Auguste, G. Jean-Louis, and S.I. McFarlane, "Shift Work and Sleep: Medical Implications and Management. Sleep Med Disord., vol. 1(2), pp. 3642, 2017. https://doi.org/10.15406/smdij.2017.01.00008

[6] S. Ganesan, M. Magee, J.E. Stone, M.D. Mulhall, A. Collins, M.E. Howard, S.W. Lockley, S.M.W. Rajaratnam, and T.L. Sletten, "The Impact of Shift Work on Sleep, Alertness and Performance in Healthcare Workers. Sci Rep., vol. 9, 4635, 2019. https://doi.org/10.1038/s41598-019-40914-x

[7] J. Ritonja, K.J. Aronson, R.W. Matthews, D.B. Boivin, and T. Kantermann, "Working Time Society consensus statements: Individual differences in shift work tolerance and recommendations for research and practice. Ind Health., vol. 57(2), pp. 201-212, 2019. https://doi.org/10.2486/indhealth.SW-5.

[8] Y. Xie, Q. Tang, G. Chen, M. Xie, S. Yu, J. Zhao, and L. Chen, "New Insights Into the Circadian Rhythm and Its Related Diseases," Front. Physiol., vol. 10, 682, 2019. https://doi.org/10.3389/fphys.2019.00682

[9] R.W. Logan, and C.A. McClung, "Rhythms of life: circadian disruption and brain disorders across the lifespan," Nat Rev Neurosci., vol. 20, pp. 49-65, 2019. https://doi.org/10.1038/s41583-018-0088-y

[10] A. Polo, S. Singh, A. Crispo, M. Russo, A. Giudice, M. Montella, G. Colonna, and S. Costantini, "Evaluating the associations between human circadian rhythms and dysregulated genes in liver cancer cells," Oncol Lett., vol. 14(6), pp. 7353-7359, 2017. https://doi.org/10.3892/ol.2017.7109. 
[11] T. Momma, H. Okayama, M. Saitou, H. Sugeno, N. Yoshimoto, Y. Takebayashi, S. Ohki, and S. Takenoshita, "Expression of circadian clock genes in human colorectal adenoma and carcinoma. Oncol Lett., vol. 14(5), pp. 5319-5325, 2017. https://doi.org/10.3892/ol.2017.6876.

[12] J. Zhao, X. Zhou, Q. Tang, R. Yu, S. Yu, Y. Long, C. Cao, J. Han, A Shi, J.J. Mao, X. Chen, and L. Chen, "BMAL1 Deficiency Contributes to Mandibular Dysplasia by Upregulating MMP3," Stem Cell Reports, vol. 10(1), pp. 180-195, 2018. https://doi.org/10.1016/j.stemcr.2017.11.017.

[13] A. Angelousi, E. Kassi, N. Nasiri-Ansari, M.O. Weickert, H. Randeva, and G. Kaltsas, "Clock genes alterations and endocrine disorders," Eur J Clin Invest., vol. 48(6), 12927, 2018 https://doi.org/10.1111/eci.12927.

[14] S. Hergenhan, S. Holtkamp, and C. Scheiermann, "Molecular Interactions Between Components of the Circadian Clock and the Immune System," J Mol Biol., vol. 432(12), pp. 3700-3713, 2020. https://doi.org/10.1016/j.jmb.2019.12.044

[15] E. H. Thorarinsdottir, E. Bjornsdottir, B. Benediktsdottir, C. Janson, T. Gislason, T. Aspelund, S.T. Kuna, A.I. Pack, and E.S. Arnardottir, "Definition of excessive daytime sleepiness in the general population: Feeling sleepy relates better to sleep-related symptoms and quality of life than the Epworth Sleepiness Scale score. Results from an epidemiological study," J Sleep Res., vol. 28(6), e12852, 2019. https://doi.org/10.1111/jsr.12852.

[16] S. M. James, K. A. Honn, S. Gaddameedhi, and H. P. A. Van Dongen, "Shift Work: Disrupted Circadian Rhythms, and Sleep-implications for Health and Well-Being," Curr Sleep Med Rep., vol. 3(2), pp. 104 112, 2017. https://doi.org/10.1007/s40675-017-0071-6.

[17] P. Afonso, M. Fonseca, and J. F. Pires, "Impact of working hours on sleep and mental health," Occup Med (Lond.)., vol. 67(5), pp. 377382, 2017. https://doi.org/10.1093/occmed/kqx054

[18] J. Ryu, K. Jung-Choi, K.-H. Choi, H.-J. Kwon, C. Kang, and H. Kim, "Associations of shift work and its duration with work-related injury among electronics factory workers in South Korea," Int. J. Environ. Res. Public Health, vol. 14(1429), 2017. https://doi.org/10.3390/ijerph14111429

[19] M. Majeed, K. Nagabhushanam, S. Arumugam, S. Majeed, and F. Ali, "Bacillus coagulans MTCC 5856 for the management of major depression with irritable bowel syndrome: a randomised, double-blind, placebo controlled, multi-centre, pilot clinical study," Food Nutr Res. vol. 62, 2018. https://doi.org/10.29219/fnr.v62.1218.

[20] M. Majeed, S. Majeed, K. Nagabhushanam, S. Arumugam, S. Natarajan, K. Beede, and F. Ali, "Galactomannan from Trigonella foenum-graecum L. seed: Prebiotic application and its fermentation by the probiotic Bacillus coagulans strain MTCC 5856," Food Sci Nutr. vol. 6(3), pp. 666-673, 2018. https://doi.org/10.1002/fsn3.606.

[21] M. Majeed, K. Nagabhushanam, A. Arumugam, S. Natarajan, S. Majeed, K. Beede, and F. Ali, "Cranberry seed fiber: a promising prebiotic fiber and its fermentation by the probiotic Bacillus coagulans MTCC 5856," Int J Food Sci Technol., vol. 53(7), pp. 1640-1647, https://doi.org/10.1111/ijfs.13747 